\title{
Leaving fibroids at caesarean section, is it safe?
}

\author{
Sireesha Yellamareddygari - Manas Chakrabarti • \\ Sudha Ravuri • Anjali Ahluwalia
}

Received: 28 April 2008 /Accepted: 25 September 2008 /Published online: 11 November 2008

(C) Springer-Verlag 2008

\begin{abstract}
Most obstetricians try to avoid myomectomy during caesarean section due to associated risks of haemorrhage. Leaving them is not entirely without complications. We report a rare case of spontaneous expulsion of a large fibroid following caesarean section.
\end{abstract}

Keywords Fibroids · Myomectomy ·

Caesarean myomectomy $\cdot$ Expulsion of fibroids

\section{Case report}

A 35-year-old woman had a booking scan which suggested a single intra-uterine pregnancy with a 6-cm fibroid in the posterior wall of the uterus. Pregnancy was uneventful in first and second trimester. A repeat scan in second trimester showed increase in size of the fibroid. She went into preterm labour at 34 weeks of gestation and had an

\footnotetext{
S. Yellamareddygari $(\square)$

Royal Lancaster Infirmary,

Flat 29, Block 2, Pointer Court Ashton Road,

Lancaster LA1 4JT, UK

e-mail: ysireesha@rediffmail.com

M. Chakrabarti $\cdot$ S. Ravuri $\cdot$ A. Ahluwalia

Obstetrics and Gynaecology,

South Manchester University Hospitals,

Manchester, UK

M. Chakrabarti

e-mail: drmanas@gmail.com

S. Ravuri

e-mail: sravuri@gmail.com

A. Ahluwalia

e-mail: anjali.ahluwalia@smuht.nwest.nhs.uk
}

emergency caesarean section for mal-presentation. The fibroid in the posterior wall was about $12 \mathrm{~cm}$ in size and was left untouched. Post-operative recovery was uneventful and she was discharged home on day 5 . She presented to gynaecology assessment unit at 6 weeks post-partum with complaints of persistent foul smelling vaginal discharge for 3 weeks and difficulty in passing urine for 2 days. She gave history of severe cramps in her pelvis 4 days prior to her presentation.

Her observations were stable and abdominal examination revealed a distended bladder, catheterisation drained $800 \mathrm{ml}$ of urine and a palpable 20-week uterus was noted. Speculum examination revealed a large globular mass in her vagina. An urgent ultrasound suggested a mass occupying the lower part of uterine cavity measuring $14 \times$ $10 \mathrm{~cm}$ with non-specific features. Magnetic resonance imaging pelvis suggested the mass to be a fibroid arising from the left posterior uterine wall above the internal os with uterine fundus clearly separate above the mass. She underwent an emergency myomectomy and a fibroid weighing $750 \mathrm{~g}$ was removed per vaginum by morcellation. Haemostasis was ensured at the base of fibroid at the end of procedure with electrocautery. Recovery was uneventful and the patient was discharged home on day 2. Speculum examination 4 weeks later revealed a completely normal looking cervix and a scan revealed no evidence of fibroid in the left uterocervical junction. Histopathology suggested a large necrotic fibroid (Fig. 1).

\section{Discussion}

Uterine fibroids are the commonest of gynaecological tumours. They are commonly dealt with a myomectomy or hysterectomy as an interval procedure. Most of women 


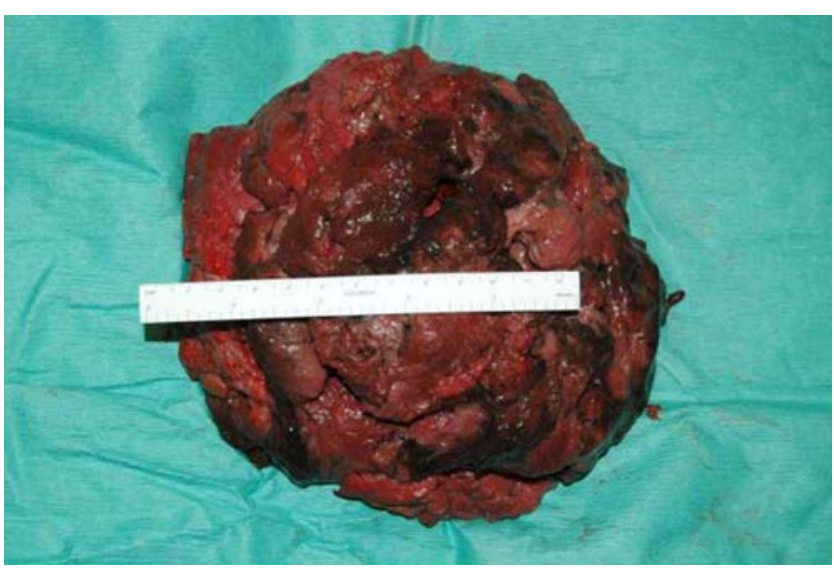

Fig. 1 Excised specimen of fibroid

with uterine fibroids complicating pregnancy will deliver vaginally but a small percentage require caesarean section.

Myomectomy during caesarean section has traditionally been discouraged because of the risk of the uncontrollable haemorrhage. Of the nine cases of caesarean myomectomy reported by Exocoutos and Rosati [1], three were complicated by severe haemorrhage necessitating hysterectomy. Ehigiegba et al. [2] studied 25 women who underwent caesarean myomectomy. They used high-dose oxytocin intra-operatively and continued its use for $12-24 \mathrm{~h}$ postoperatively. Their patients had greater blood loss $(876 \pm$ $312 \mathrm{ml})$ and higher rate (20\%) blood transfusion. Seventytwo percent of patients had post-operative morbidity.

Over the last decade, there has been a trend towards caesarean myomectomy. Apart from risk of severe haemorrhage, there appears to be no other absolute contraindication to routine caesarean myomectomy. Roman and Tabsh [3] in their retrospective study concluded that in selected patients, myomectomy during caesarean section does not appear to result in an increased risk of intra-partum or short-term post-partum morbidity. This view was supported by Kaymac et al. [4] in their retrospective case control study. In another retrospective case control study, Brown et al. [5] compared 16 women with uterine fibroids in pregnancies who were treated by caesarean myomectomy, with 16 women without uterine fibroids who had caesarean section during the same period. They did not find any difference in the blood loss or morbidity between the two groups. They concluded that caesarean myomectomy may be safe in highly selected cases when done by experienced team.

Leaving fibroids untouched during myomectomy has shown to be associated with complications in few of the studies. Hasan et al. [6] noted a high incidence of hysterectomy for post-partum haemorrhage. An increased incidence of post-partum sepsis has been noted by Davis et al. [7] in cases where the myomas were not removed.
Fibroids in puerperium can lead to sub-involution, may undergo red degeneration and become a focus for infection.

Caesarean myomectomy is probably not indicated in asymptomatic, small fibroids, or those in intramural location. It appears to be safe and best reserved for patients with symptomatic fibroids which are in sub-serosal or pedunculated location and in those affecting the lower uterine segment. Ehigiegba et al. [2] removed single fibroids when they were well above $5 \mathrm{~cm}$; in their study, most of patients had multiple fibroids which were intramural in location. They did not remove any posteriorly placed fibroids.

Proponents have used varying techniques to decrease the blood loss during caesarean myomectomy. Most common practise was to use high-dose oxytocin intra- and postoperatively. An infiltrate of oxytocin diluted with an equal volume of normal saline injected into the capsule overlying the fibroid was used by Brown et al. [5] to help achieve haemostasis. Sapmaz et al. [8] conducted a randomised controlled study to compare the efficacy of bilateral ascending uterine artery ligation vs. tourniquet use for haemostasis in caesarean myomectomy. Uterine artery ligation appears to be alternative method for treating pregnant women with uterine leiomyomas, who are undergoing caesarean section; it was shown to minimise post-partum blood loss and significantly reduce the necessity of future surgery [9].

Rare cases expulsion of fibroids has been reported following uterine artery embolisation [10]. Extensive literature search did not reveal spontaneous expulsion of fibroids following caesarean section. Our case report is interesting as it is the first in English literature to note expulsion of such a large single fibroid. Though it has been traditionally taught that leaving uterine fibroids at caesarean is a safe option to prevent intra-operative complications, it may be not be safe to do so in all cases as shown in our case report.

\section{References}

1. Exacoustos C, Rosati P (1993) Ultrasound diagnosis of myomas and complications in pregnancy. Obstet Gynecol 82(1):97-101

2. Ehigiegba AE, Ande AB, Ojobo SI (2001) Myomectomy during cesarean section. Int J Gynaecol Obstet 75(1):21-5

3. Roman AS, Tabsh KM (2004) Myomectomy at time of caesarean section: a retrospective cohort study. BMC Pregnancy Childbirth 4:14

4. Kaymac, Ustunyurt E, Okayay RE, Kalyoncu S, Molla Mahmutoglu L (2005) Myomectomy during caesarean section. Int J Gynecol Obstet 89:90-93

5. Brown D, Fletcher HM, Myrie MO, Reid M (1999) Caesarean myomectomy - a safe procedure. A retrospective case controlled study. J Obstet Gynaecol 19(2):139-141

6. Hasan F, Arumigam K, Sivanesaratnam V (1990) Uterine leiomyomata in pregnancy. Int J Gynecol Obstet 34:45-48 
7. Davis JL, Ray-Mazumder S, Hobel CJ, Baley K, Sassoon D (1990) Uterine leiomyomas in pregnancy: a prospective study. Obstet Gynecol 75:41-44

8. Sapmaz E, Celik H, Altungül A (2003) Bilateral ascending uterine artery ligation vs. tourniquet use for hemostasis in cesarean myomectomy. A comparison. J Reprod Med 48 (12):950-954
9. Liu WM, Wang PH, Tang WL, Wang IT, Tzeng CR (2006) Uterine artery ligation for treatment of pregnant women with uterine leiomyomas who are undergoing cesarean section. Fertil Steril 86(2):423-428

10. Laverge F, D'Angelo A, Davies NJ, Wood A, Amso NN (2003) Spontaneous expulsion of three large fibroids after uterine artery embolization. Fertil Steril 80:450-452 